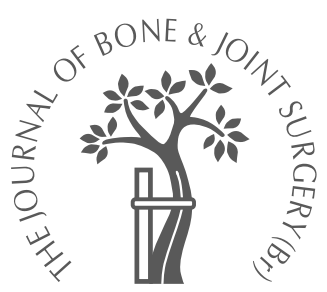
A. Whaley,
B. F. Morrey,
R. Adams

From the Mayo

Clinic, Rochester,

Minnesota, USA
A. Whaley, MD, Consultant Christus Santa Rosa, Medical Tower 1, 2829 Babcock Road, Su.700, San Antonio, Texas 78229, USA.

B. F. Morrey, MD, Professor of Orthopaedics

R. Adams, MA, RPA

Department of Orthopaedic

Surgery, Mayo Clinic, 200

First Street SW, Rochester,

Minnesota 55905, USA.

Correspondence should be sent to Professor B. F.

Morrey.

(C)2005 British Editorial

Society of Bone and

Joint Surgery

doi:10.1302/0301-620X.87B1.

$14543 \$ 2.00$

$J$ Bone Joint Surg $[\mathrm{Br}]$

2005;87-B:47-53.

Received 29 April 2003

Accepted after revision

4 March 2004

\title{
Total elbow arthroplasty after previous resection of the radial head and synovectomy
}

We examined the effects of previous resection of the radial head and synovectomy on the outcome of subsequent total elbow arthroplasty in patients with rheumatoid arthritis.

Fifteen elbows with a history of resection and synovectomy were compared with a control group of patients who had elbow arthroplasty with an implant of the same design. The mean age in both groups was 63 years. In the study group, resection of the radial head and synovectomy had been undertaken at a mean of 8.9 years before arthroplasty. The mean radiological follow-up for the $\mathbf{1 3}$ available patients in the study group was $\mathbf{5 . 8 9}$ years (0.3 to 11.0) and in the control group was 6.6 years $(2.2$ to 12.6$)$. There were no revisions in either group. The mean Mayo elbow performance score improved from 29 to 96 in the study group, with similar improvement in the control group (28 to 87). The study group had excellent results in 13 elbows and good results in two. The control group had excellent results in seven and good results in six.

Our experience indicates that previous resection of the radial head and synovectomy are not associated with an increased rate of revision following subsequent arthroplasty of the elbow. However, there was a higher rate of complication in the study group compared with the control group.

The challenge for orthopaedic surgeons treating patients with rheumatoid arthritis of the elbow is to achieve a painless, stable, and functional elbow. The initial management consists of medical treatment to control synovitis. If this fails, various surgical options can be considered. Several reports have shown good results following resection of the radial head and synovectomy. ${ }^{1-13}$ The results of total elbow arthroplasty in these patients have also been good. ${ }^{14-21}$ Data are limited on the effect of a previous resection of the radial head and synovectomy on subsequent total elbow arthroplasty. $^{22}$ Our aim, therefore, was to compare the outcome of total elbow arthroplasty with and without a history of previous surgery.

\section{Patients and Methods}

We used the joint replacement computer database at our institution to identify those patients with rheumatoid arthritis who had been treated by total elbow arthroplasty (Coonrad Morrey; Zimmer, Warsaw, Indiana) and who had a previous excision of the radial head and synovectomy. To detect more accurately the effect, if any, of the previous surgery, a control group was identified. This was developed from a database of patients with rheumatoid arthritis who had been treated using the same prosthesis during the same period of time. None had a previous resection of the radial head and synovectomy and they were matched by gender, side of involvement, and age. There was a minimum follow-up of two years for both groups. We retrospectively reviewed the records and radiographs of both groups. Additional contact was attempted in all patients. The Mayo elbow performance score (MEPS) was used to assess pain, movement, stability, and daily function as has been described previously. ${ }^{18,21,23,24}$ A result was considered to be satisfactory if an excellent or good rating was attained with the MEPS. The pre-operative radiographs were reviewed and categorised in accordance with the Mayo classification of rheumatoid involvement of the elbow. ${ }^{18}$ The post-operative and latest radiographs were reviewed in order to identify radiolucent lines and evidence of loosening.

A total of 16 elbows was identified in 15 patients who had undergone a total elbow arthroplasty having previously undergone a resection of the radial head and synovectomy. We excluded one patient with an elbow arthroplasty who died 88 days after the operation of 


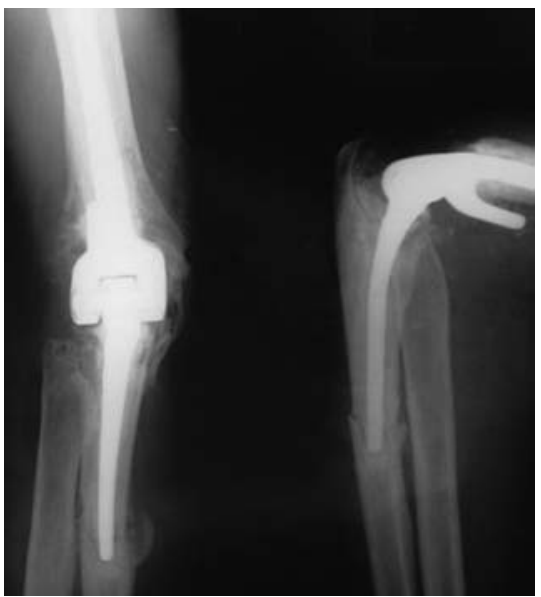

Fig. 1a

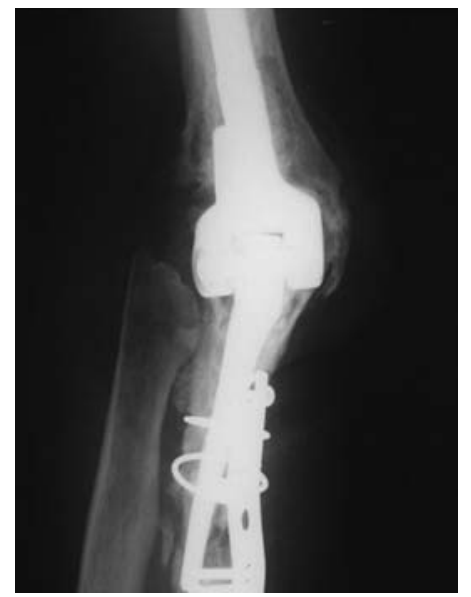

Fig. 1b

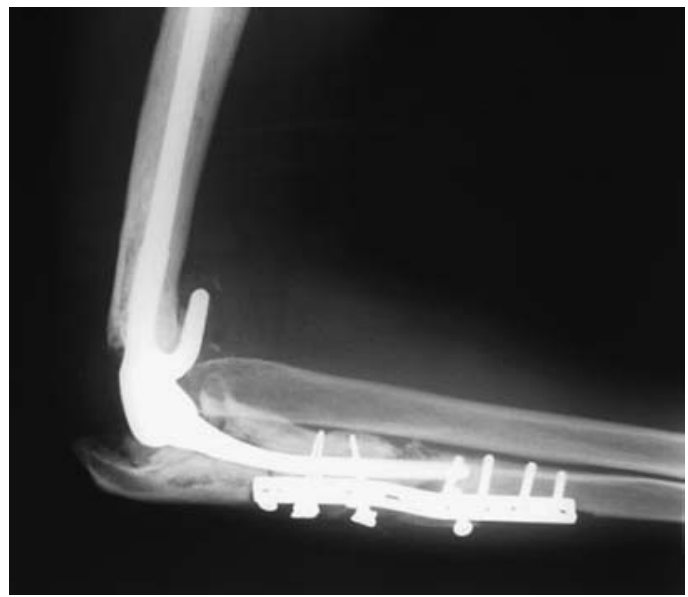

Fig. 1c

Radiographs (AP and lateral) showing a periprosthetic fracture of the ulna four years after total elbow arthroplasty (a). Subsequent radiographs, b) AP and c) lateral showing union after fixation

Table I. Details of the radiological findings in the study group

\begin{tabular}{|c|c|c|c|c|c|}
\hline Case & $\begin{array}{l}\text { Radiological } \\
\text { follow-up (yrs) }\end{array}$ & $\begin{array}{l}\text { Mayo classification of } \\
\text { rheumatoid arthritis }^{18}\end{array}$ & \multicolumn{2}{|l|}{ Radiolucency } & Bone graft anterior flange \\
\hline 1 & 0.3 & $3 A$ & None & None & Incorporated bone graft \\
\hline 3 & 7.6 & 2 & None & None & Incorporated bone graft \\
\hline 4 & 1.4 & 4 & None & None & Incorporated bone graft \\
\hline 6 & None & 4 & Deceased & Deceased & Deceased \\
\hline 7 & 3.6 & 4 & None & None & Incorporated bone graft \\
\hline 8 & 6.0 & $3 \mathrm{~A}$ & None & $\begin{array}{l}1 \mathrm{~mm} \text { lucency } \\
\text { proximal } 1 / 3 \text { ulna }\end{array}$ & Incorporated bone graft \\
\hline 9 & 10.0 & 2 & None & None & Incorporated bone graft \\
\hline 13 & 5.3 & 2 & None & $\begin{array}{l}\text { Lysis at tip and } \\
\text { around precoat } \\
\text { proximally }\end{array}$ & Incorporated bone graft \\
\hline 14 & None & $3 \mathrm{~A}$ & Refused & Refused & Refused \\
\hline 15 & 9.3 & 3B & None & None & Incorporated bone graft \\
\hline
\end{tabular}

causes unrelated to the elbow surgery. Thus, there were 15 elbows in 14 patients in the study group.

The involved side was the right in seven, the left in six and one patient had undergone bilateral elbow arthroplasties. The mean age at the time of arthroplasty was 62.8 years (47 to 74). Only five of the 15 elbows had undergone previous resection of the radial head and synovectomy at our institution at a mean of 8.5 years (0.6 to 30.9$)$ before the replacement. The remaining ten elbows had the initial surgery elsewhere at a mean of 9.1 years (1 to 24$)$. Altogether, the resection of the radial head and synovectomy had been undertaken at a mean of 8.9 years $(0.6$ to 30.9$)$ before the total elbow arthroplasty. The indication for elbow arthro- plasty was progression of the disease in all patients with pain and limitation of function. One patient died of unrelated causes. The elbows were in place and functioning at the time of death.

The control group of 15 elbows was identified from a series of consecutive procedures performed for the same condition during the same period. This group was also matched by gender and duration of surveillance and comprised of 11 women and four men. The involved side was the right in eight and the left in seven patients. The mean age of the time of arthroplasty for this group was 63.6 years (44 to 81 ). The indication for total elbow arthroplasty was progression of disease in all patients with pain and limita- 


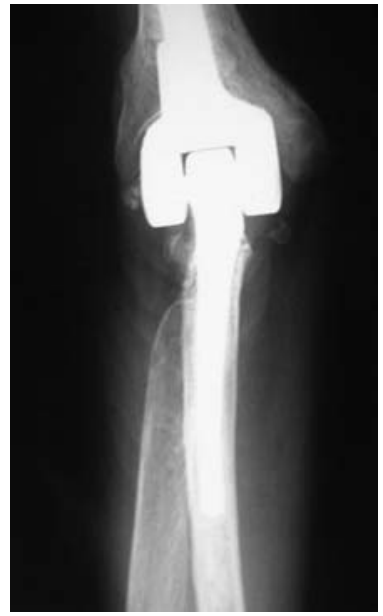

Fig. 2a

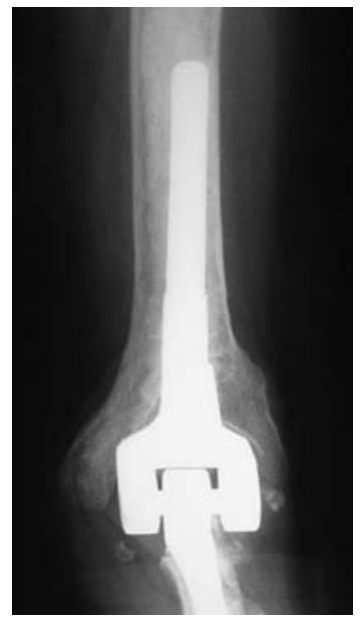

Fig. $2 b$

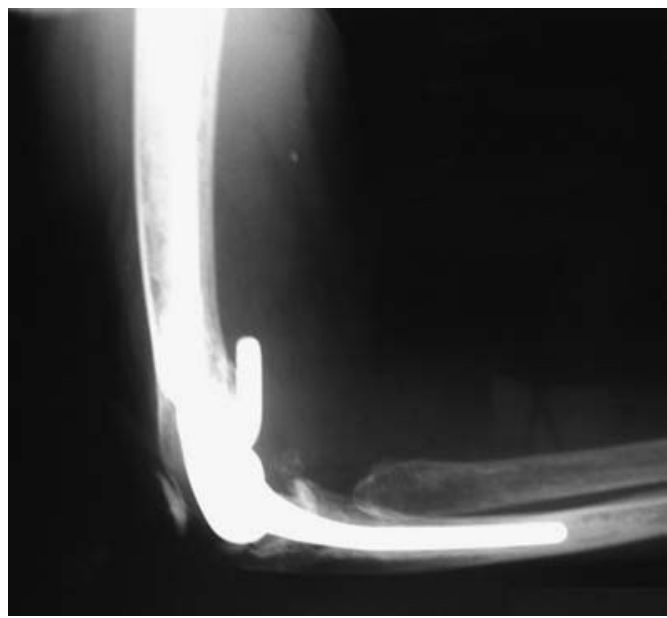

Fig. 2c

Radiographs a) and b) AP and c) lateral 8.5 years after a two-stage bushing exchange and rearticulation for acute deep infection.

Table II. Details of the clinical outcome in both groups showing range of movement in degrees $\left({ }^{\circ}\right)$, pain and stability

\begin{tabular}{|c|c|c|c|c|c|c|c|c|c|c|c|c|}
\hline \multirow[b]{2}{*}{ Group } & \multicolumn{2}{|c|}{ Extension } & \multicolumn{2}{|l|}{ Flexion } & \multicolumn{2}{|c|}{ Pronation } & \multicolumn{2}{|c|}{ Supination } & \multicolumn{2}{|l|}{ Pain } & \multicolumn{2}{|l|}{ Stability } \\
\hline & Pre-op & Post-op & Pre-op & Post-op & Pre-op & Post-op & Pre-op & Post-op & Pre-op & Post-op & Pre-op* & Post-op \\
\hline \multicolumn{13}{|l|}{ Study } \\
\hline 1 & 20 & 20 & 140 & 145 & 60 & 85 & 70 & 85 & Severe & None & Mild & Stable \\
\hline 2 & 45 & 60 & 130 & 110 & 0 & 30 & 70 & 30 & Moderate & None & Moderate & Stable \\
\hline 3 & 30 & 0 & 130 & 110 & 60 & 70 & 70 & 70 & Mod-sev & None & Mild & Stable \\
\hline 4 & 0 & 30 & 80 & 130 & 80 & 0 & 90 & 0 & Severe & None & $\begin{array}{l}\text { Severe ML } \\
\text { lax }\end{array}$ & Stable \\
\hline 5 & 25 & 60 & 125 & 130 & 20 & 90 & 70 & 0 & Severe & Mild, rare & Mild ML lax & Stable \\
\hline 6 & 30 & 0 & 130 & 120 & 60 & 90 & 70 & 90 & Severe & None & Severe lax & Stable \\
\hline 7 & 0 & 0 & 130 & 120 & 50 & 90 & 50 & 90 & Severe & None & Unstable & Stable \\
\hline 8 & 40 & 30 & 90 & 120 & 60 & 90 & 50 & 90 & Severe & None & Mild & Stable \\
\hline 9 & 30 & 30 & 115 & 120 & 60 & 90 & 60 & 90 & Severe & None & Moderate & Stable \\
\hline 10 & 20 & 30 & 130 & 130 & 30 & 90 & 30 & 90 & Severe & Mild, rare & Mild & Stable \\
\hline 11 & 30 & 20 & 120 & 130 & 70 & 90 & 70 & 90 & Severe & None & Moderate & Stable \\
\hline 12 & 0 & 45 & 140 & 130 & 80 & 90 & 80 & 60 & Severe & None & Severe & Stable \\
\hline 13 & 70 & 30 & 110 & 130 & 75 & 90 & 75 & 90 & Severe & None & Stable & Stable \\
\hline 14 & 30 & 30 & 120 & 120 & 60 & 90 & 60 & 60 & Severe & None & Stable & Stable \\
\hline \multicolumn{13}{|c|}{ Control } \\
\hline 1 & 40 & 0 & 120 & 130 & 40 & 90 & 30 & 90 & Severe & None & Mild & Stable \\
\hline 2 & 40 & 30 & 120 & 130 & 65 & 90 & 70 & 90 & Severe & Mild & Stable & Stable \\
\hline 3 & 45 & 30 & 130 & 130 & 60 & 60 & 70 & 60 & Severe & Mild & Stable & Stable \\
\hline 4 & 70 & 30 & 120 & 130 & 30 & 60 & 30 & 60 & Moderate & None & Stable & Stable \\
\hline 5 & 75 & 60 & 95 & 130 & 10 & 30 & 10 & 30 & Severe & Mild & Stable & Stable \\
\hline 6 & 35 & 60 & 125 & 90 & 60 & 30 & 70 & 30 & Severe & Mild & Mild & Stable \\
\hline 7 & 55 & 35 & 130 & 125 & 60 & 60 & 45 & 30 & Severe & None & Unstable & Stable \\
\hline 8 & 35 & 60 & 115 & 120 & 15 & 60 & 15 & 60 & Severe & None & Moderate & Stable \\
\hline 9 & 10 & 10 & 135 & 135 & 40 & 40 & 60 & 60 & Severe & Mild & Mild & Stable \\
\hline 10 & 25 & 25 & 125 & 135 & 70 & 80 & 70 & 70 & Severe & None & Mild & Stable \\
\hline 11 & 30 & 90 & 100 & 120 & 40 & 90 & 40 & 90 & Severe & None & Moderate & Stable \\
\hline 12 & 25 & 30 & 120 & 130 & 55 & 90 & 0 & 30 & Severe & Mild & Mild & Stable \\
\hline 13 & 10 & 30 & 135 & 120 & 50 & 90 & 60 & 90 & Severe & Mild & Stable & Stable \\
\hline 14 & 10 & 40 & 120 & 110 & 70 & 45 & 70 & 45 & Severe & None & Mild & Stable \\
\hline 15 & 50 & 30 & 115 & 130 & 50 & 90 & 60 & 90 & Severe & None & Stable & Stable \\
\hline
\end{tabular}

\footnotetext{
* ML, mediolateral
} 
Table III. Details of the radiological results in the control group

\begin{tabular}{|c|c|c|c|c|c|c|}
\hline \multirow[b]{2}{*}{ Case } & \multirow{2}{*}{$\begin{array}{l}\text { Radiological follow-up } \\
\text { (yrs) }\end{array}$} & \multirow{2}{*}{$\begin{array}{l}\text { Time from TEA* } \\
\text { to death (yrs) }\end{array}$} & \multirow{2}{*}{$\begin{array}{l}\text { Mayo classification of } \\
\text { rheumatoid arthritis }\end{array}$} & \multicolumn{2}{|l|}{ Radiolucency } & \multirow{2}{*}{$\begin{array}{l}\text { Bone graft anterior- } \\
\text { flange }\end{array}$} \\
\hline & & & & Humeral component & Ulnar component & \\
\hline 1 & 5.2 & & $3 A$ & None & None & Incorporated \\
\hline 3 & 2.2 & & 2 & None & None & Incorporated \\
\hline 4 & 12.6 & & $3 A$ & $\begin{array}{l}\text { None, mild wear of } \\
\text { bushing }\end{array}$ & None & Incorporated \\
\hline 5 & 2.6 & & $3 A$ & None & None & Incorporated \\
\hline 6 & 5.2 & 5.8 & $3 A$ & None & None & Incorporated \\
\hline 7 & 10.5 & & 2 & None & None & Incorporated \\
\hline 8 & 11.0 & & 4 & None & None & Incorporated \\
\hline 9 & 3.0 & 6.6 & $3 A$ & None & None & Incorporated \\
\hline 10 & 7.8 & & 2 & None & Lucency at tip & Incorporated \\
\hline 11 & 6.2 & & $3 A$ & None & None & Incorporated \\
\hline 12 & 2.5 & & 2 & None & None & Incorporated \\
\hline 13 & 5.2 & & 2 & None & $\begin{array}{l}\text { Proximal ulnar } \\
\text { lucency }\end{array}$ & Incorporated \\
\hline 14 & 10.0 & & $3 A$ & None, wear of bushing & None & Incorporated \\
\hline 15 & 7.0 & & $3 B$ & None & None & Incorporated \\
\hline
\end{tabular}

* TEA, total elbow arthroplasty

tion of function. Two patients had died at 5.8 and 6.6 years respectively after the arthroplasty. Both elbows were in place and functioning at the time of death.

\section{Results}

Current information was obtained on all patients until death (Tables I and II). In the study group, one patient refused further radiography but clinical assessment was good. A second patient died (at three months) after the initial procedure but before any follow-up could be carried out. The mean clinical follow-up for the 14 available patients was 6 years ( 2 to 9.2). In the control group, the mean clinical follow-up was 7.4 years (2.0 to 15.1 ; Table III). The mean radiological follow-up of the study group was 5.8 years $(0.3$ to 11.0$)$ compared with that in the control group of 6.6 years (2.2 to 12.6). There were no revisions in either group.

Complications and re-operations. In the study group there were five complications in five arthroplasties (Table IV). One patient had an undisplaced intra-operative fracture of the ulna which was recognised and treated by cerclage wiring. The same patient developed a spontaneous undisplaced fracture of the lateral epicondyle post-operatively which was successfully treated by splinting. This patient did well and had a painless and stable elbow at 4.3 years. A second patient subsequently fell and sustained a periprosthetic fracture of the ulna, which was treated elsewhere by open reduction, internal fixation, and retention of the arthroplasty. This patient was also doing well at 8.6 years (Fig. 1). A third patient developed an acute deep infection which was diagnosed two months after the initial procedure. This patient was successfully treated by acute debridement and a two-stage bushing exchange and articulation. At 8.5 years there was no evidence of loosening or recurrent infection (Fig. 2). A fourth patient sustained an intra-operative fracture of the olecranon when the ulnar trial component was being inserted. This was treated by tension-band fixation.
At 5.4 years a fibrous union was present. At the last clinical follow-up at 8.2 years, extension lacked $45^{\circ}$ but the patient had a stable and painless elbow with extension strength of $75 \%$. The fifth patient developed a superficial infection two weeks after the arthroplasty which was settled with oral antibiotics for three weeks and there were no further complications.

In the control group, there were two complications in two arthroplasties (Table IV). One patient developed dehiscence of the wound two weeks after the initial arthroplasty, and was treated by irrigation and debridement and primary closure without further complications. Another sustained an intra-operative fracture of the lateral epicondyle which was minimally displaced. This fracture was deemed to be stable at the time of surgery and was not fixed. This patient was doing well with a painless, fibrous union and a stable elbow 15.1 years later. While there is a numerical difference in the number of complications in the two groups, the difference is not statistically significant (chi-squared test was not significant). Assessment of each complication does not suggest that there is an increased incidence of problems which may be attributable to the previous surgery or absence of the radial head except for the observation of one deep and one superficial infection in the study group.

Radiological findings. The pre-operative radiographs were reviewed by the authors and graded in accordance with the Mayo classification of rheumatoid involvement ${ }^{18}$ (Table I). In type 1 there is a normal joint with synovitis and/or osteoporosis. In type 2 there is joint narrowing and in type 3 early (A) or lateral (B) architectural changes in the joint. In type 4 the joint is grossly destroyed (mutilans). In the study group there were no type- 1 elbows, five type- 2 , four type$3 \mathrm{~A}$, one type- $3 \mathrm{~B}$, and five type- 4 (Table I). In the control group, there were no type-1, five type-2, eight type-3A, one type-3B, and one type-4 elbows (Table III).

The available radiographs were reviewed for the presence of radiolucencies or evidence of loosening (Table I). In 
the study group, no humeral component had a radiolucency. Two elbows had non-progressive radiolucencies around less than $25 \%$ of the precoat area of the ulnar component only (Fig. 3). One elbow had an area of lysis, $4 \mathrm{~mm}$ wide, at the tip of the ulnar component and one an area, 3 $\mathrm{mm}$ wide, at the mid-portion of the precoat; neither patient had pain. All bone grafts had incorporated behind the flange of the implant at the last follow-up. None of these elbows was loose or considered to have failed. In the control group, no humeral components had a radiolucency. One had an area of lysis $3 \mathrm{~mm}$ wide, at the tip of the ulnar component and one had an incomplete lucency involving the proximal half of the ulna. Two other elbows in the control group were noted to have bushing wear as defined by Gill and Morrey ${ }^{21}$ which did not affect function (Fig. 4). In all elbows of the control group the bone graft behind the flange had incorporated. Considering all the radiological data of lucency and bushing wear, five events were recorded in the study and four in the control groups. There were no revisions in either group.

The Mayo elbow performance scale (MEPS). The MEPS score for the study group improved from a pre-operative mean of 29 (15 to 65) to a mean of 96 (80 to 100) at the most recent follow-up. For the control group, the score improved from a pre-operative mean of 28 (20 to 45$)$ to a mean of 86 (65 to 100) at the most recent follow-up. Subjectively, there were 13 excellent and two good results in the study group and six excellent, seven good, and two fair results in the control group. Both patients with fair results received this designation because they had limited movement. These two patients otherwise had stable elbows with no pain and had good function.

In both groups, all patients reported that their elbow was stable at the final follow-up and all expressed satisfaction with the procedure. The range of movement improved slightly in both groups. In the study group, two patients reported having mild pain compared with eight in the control group.

\section{Discussion}

Resection of the radial head and synovectomy is a wellestablished surgical procedure used in the management of rheumatoid arthritis which has failed to respond to conservative treatment. The frequency of indications for this procedure are decreasing because of the widespread use and effectiveness of medical treatment. Nonetheless, there are limited studies which have assessed the effect of previous excision of the radial head and synovectomy on the outcome following subsequent total elbow arthroplasty.

The indications for synovectomy and excision of the radial head include uncontrolled, painful synovitis and limitation of elbow function which have failed to respond to medical management. ${ }^{1,13}$ Synovectomy alone does not reliably restore movement, and therefore has few indications in patients with advanced disease. Most results reported in the literature have shown deterioration with time..$^{2,5-7}$ The

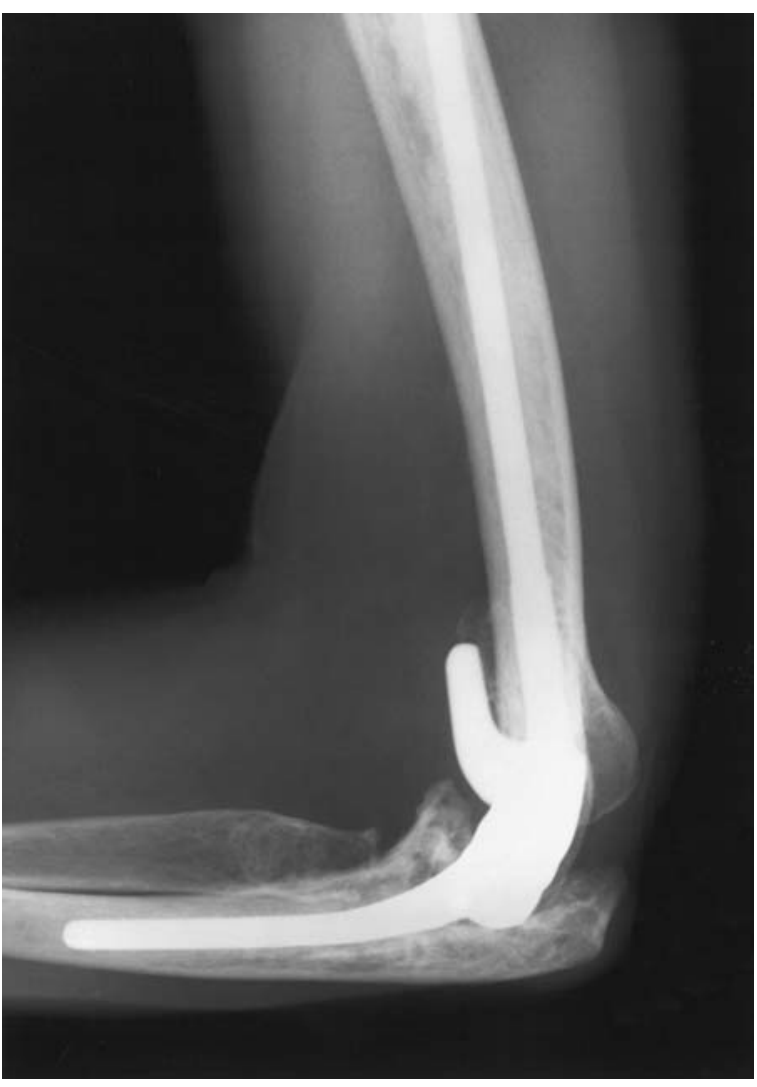

Fig. 3

Lateral radiograph showing a subtle non-progressive lucency at the proximal third of the ulnar component five years after arthroplasty.

fact that the mean duration of time in our study group from resection and synovectomy to arthroplasty was 8.9 years correlates with a deterioration of function with time after this procedure. For those patients in whom the disease has progressed after resection and synovectomy, total elbow arthroplasty is the most reliable remaining surgical option.

In several studies ${ }^{14-20}$ the results of total elbow arthroplasty in patients with rheumatoid arthritis have been satisfactory. Gill and Morrey ${ }^{21}$ reported the long-term results of the use of this same semi-constrained prosthesis (CoonradMorrey). In that study, the survivorship of the prosthesis in rheumatoid patients was $94.4 \%$ at five and $92.4 \%$ at ten years. In the current study there was radiological evidence of wear and bone remodelling in both groups, which is to be expected. However, no patients had a revision of either the humeral or ulnar component at the last follow-up (study group mean 6.2 years, control group mean 9.1 years). Gill and Morrey ${ }^{21}$ reported the MEPS to be good or excellent in $86 \%$ of patients. In our study $100 \%$ of the study group and $88 \%$ of the control group had excellent or good results using the same criteria. There was no difference in the outcome between the two groups. 


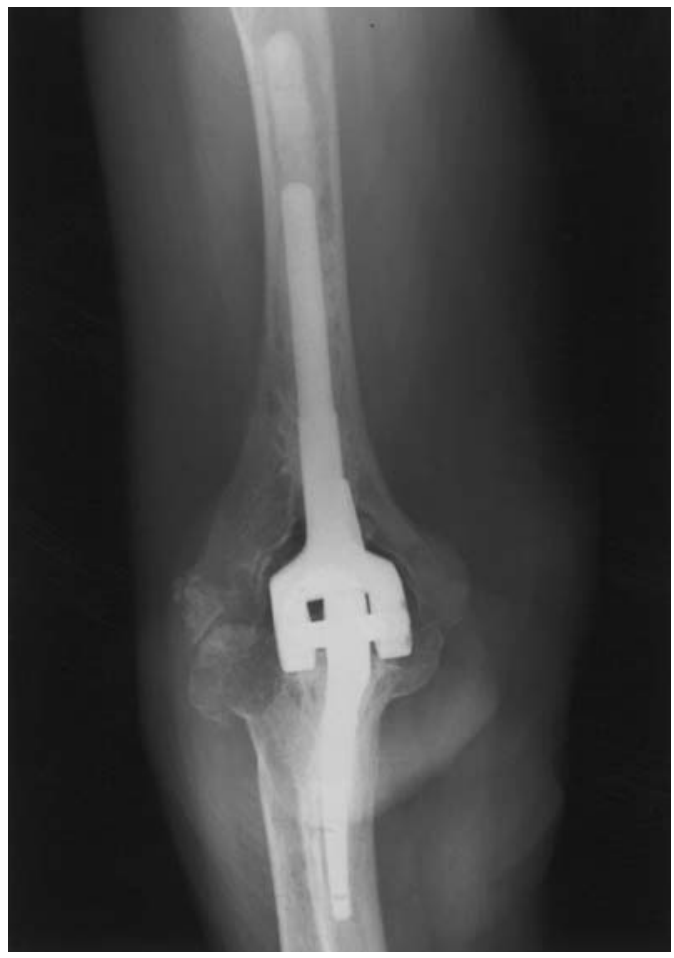

Fig. 4a

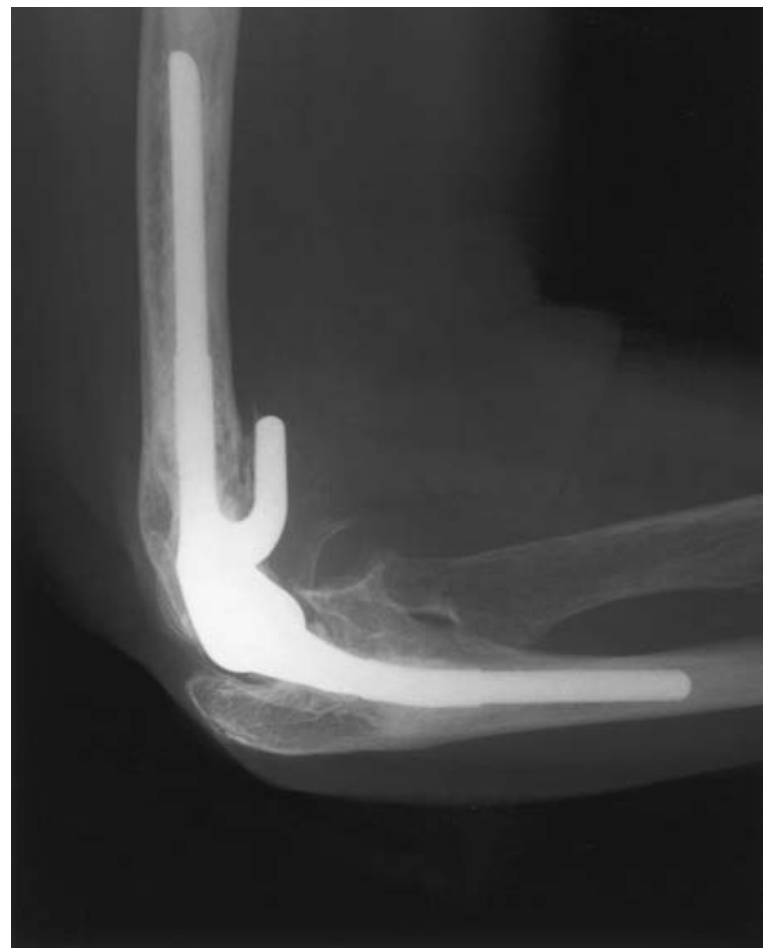

Fig. 4b

Radiographs a) AP and b) lateral showing the outcome after 11 years with some wear of the articulation but no lysis.

Table IV. Details of complications and re-operations in both groups

\begin{tabular}{|c|c|c|}
\hline Group & Complications & Time to follow-up (yrs) \\
\hline \multicolumn{3}{|l|}{ Study } \\
\hline 1 & Intra-operative fracture of ulna and lateral epicondyle & 4.3 \\
\hline 2 & $\begin{array}{l}\text { Distal ulnar periprosthetic fracture, treated with ORIF* of ulna with } \\
\text { retention of components }\end{array}$ & 8.6 \\
\hline 3 & $\begin{array}{l}\text { Acute deep infection, treated by irrigation and debridement with retention } \\
\text { of components (except bushings and pin) }\end{array}$ & 8.5 \\
\hline 4 & $\begin{array}{l}\text { Intra-operative olecranon fracture, with later asymptomatic diastasis at the } \\
\text { fracture site noted }\end{array}$ & 8.2 \\
\hline 5 & Superficial wound infection treated with oral antibiotics & 1.3 \\
\hline \multicolumn{3}{|c|}{ Control } \\
\hline 1 & $\begin{array}{l}\text { Wound dehiscence at the distal aspect of the wound, treated by irrigation, } \\
\text { debridement and primary closure with retention of components (no sign of } \\
\text { infection noted) }\end{array}$ & 5.2 \\
\hline 2 & Intra-operative fracture of the lateral epicondyle & 15.1 \\
\hline
\end{tabular}

* ORIF, open reduction and internal fixation

To the best of our knowledge, there has been only one other study published in the English literature which has examined the results of elbow arthroplasty after previous resection of the radial head and synovectomy in patients with rheumatoid arthritis. Using a non-constrained implant, Schemitsch et $\mathrm{al}^{22}$ compared two groups of rheumatoid patients with and without this previous surgery. The mean time from the resection and synovectomy to arthroplasty was six years and the mean follow-up was four years. They concluded that arthroplasty following a previ- ous resection and synovectomy using a Capitellocondylar (Howmedica, Rutherford, New Jersey) unlinked arthroplasty was technically demanding and the results at two years were inferior compared with those without previous surgery. This difference was primarily due to instability and they recommended that if the stability of the components cannot be accomplished at the time of surgery, a more constrained device should be used. In our study, although there were no problems of stability using a linked implant, there were more complications in the study group. 
In summary, radial head resection and synovectomy did not have a measurable detrimental effect on the outcome after subsequent elbow arthroplasty. Our results are similar to those reported by Gill and Morrey ${ }^{21}$ and Morrey and Adams ${ }^{18}$ in patients with rheumatoid arthritis using the same prosthesis. There was a higher rate of complications in the study group, but this did not appear adversely to affect the long-term functional outcome.

The author or one or more of the authors have received or will receive benefits for personal or professional use from a commercial party related directly or indirectly to the subject of this article.

\section{References}

1. Boyd AD, Thornhill TS. Surgical treatment of the elbow in rheumatoid arthritis Hand Clin 1989;5:645-55

2. Brumfield RH, Resnick CT. Synovectomy of the elbow in rheumatoid arthritis. $J$ Bone Joint Surg [Am] 1985;67-A:16-20.

3. Eichenblat $\mathbf{M}$, Hass $\mathbf{A}$, Kessler I. Synovectomy of the elbow in rheumatoid arthritis. J Bone Joint Surg [Am] 1982;64-A:1074-8.

4. Ferlic DC, Patchett CE, Clayton ML, et al. Elbow synovectomy in rheumatoid arthritis: long-term results. Clin Orthop 1987;220:119-25.

5. Summers GC, Taylor AR, Webley M. Elbow synovectomy and excision of the radial head in rheumatoid arthritis: a short term palliative procedure. J Rheumatology 1988; 15:566-9.

6. Ferlic DC, Patchett CE, Clayton ML, Freeman AC. Elbow synovectomy in rheumatoid arthritis. Clin Orthop 1987;220:119-25.

7. Vahvanen V, Eskola A, Peltonen J. Results of elbow synovectomy in rheumatoid arthritis. Arch Orthop Trauma Surg 1991;110:151-4.

8. Lonner JH, Stuchin SA. Synovectomy, radial head excision, and anterior capsula release in stage III inflammatory arthritis of the elbow. J Hand Surg [Am]1997;22: 279-85.

9. Linclau LA, Winia WP, Korst JK. Synovectomy of the elbow in rheumatoid arthritis Acta Orthop Scand 1983;54:935-7.
10. Porter BB, Richardson C, Vainio K. Rheumatoid arthritis of the elbow: the results of synovectomy. J Bone Joint Surg [Br] 1974;56-B:427-37.

11. Copeland SA, Taylor JG. Synovectomy of the elbow in rheumatoid arthritis: the place of excision of the head of the radius. J Bone Joint Surg [Br] 1979;61-B:69-73.

12. Marmor L. Surgery of the rheumatoid elbow: follow-up study on synovectomy combined with radial head excision. J Bone Joint Surg [Am] 1972;54-A:573-8.

13. Saito T, Koshinon T, Okamoto R, Horiuchi S. Radical synovectomy with muscle release for the rheumatoid elbow. Acta Orthop Scand 1986;57:71-3.

14. Lyall HA, Cohen B, Clatworthy M, Constant CR. Results of the Souter-Strathclyde total elbow arthroplasty in patients with rheumatoid arthritis. J Arthroplasty 1994;9: 279-84

15. Pritchard RW. Total elbow joint arthroplasty in patients with rheumatoid arthritis Semin Arthritis Rheum 1991;21:24-9.

16. Sjoden GOJ, Blomgren GGA, Lindgren JU. The Souter total elbow replacement in rheumatoid arthritis. Scand J Rheumatol 1988;17:219-22.

17. Kudo H, Iwano K. Total elbow arthroplasty with a non-constrained surface-replacement prosthesis in patients who have rheumatoid arthritis. J Bone Joint Surg [Am] 1990;72-A:355-62.

18. Morrey BF, Adams RA. Semiconstrained arthroplasty for the treatment of rheumatoid arthritis of the elbow. J Bone Joint Surg [Am] 1992;74-A:479-90.

19. Ewald FC, Simmons ED, Sullivan JA, et al. Capitellocondylar total elbow replacement in rheumatoid arthritis: long-term results. J Bone Joint Surg [Am] 1993;75-A 498-507

20. Kasten MD, Skinner HB. Total elbow arthroplasty: an 18-year experience. Clin Orthop 1993;290:177-88.

21. Gill DRJ, Morrey BF. The Coonrad-Morrey total elbow arthroplasty in patients who have rheumatoid arthritis: a ten to fifteen year follow up study. J Bone Joint Surg [Am] 1998:80-A:1327-35

22. Schemitsch EH, Ewald FC, Thornhill TS. Results of total elbow arthroplasty after excision of the radial head and synovectomy in patients who had rheumatoid arthritis. J Bone Joint Surg [Am] 1996;78-A:1541-7.

23. King GJW, Adams RA, Morrey BF. Total elbow arthroplasty: revision with use of a non-custom semiconstrained prosthesis. J Bone Joint Surg [Am] 1997;79-A:394-400.

24. Morrey BF, Adams RA. Semiconstrained elbow replacement for distal humeral nonunion. J Bone Joint Surg [Br] 1995;77-B:67-72. 